\title{
Self-similar Mesocrystals Form Via Interface-Driven Nucleation and Assembly and Dissolve by Particle Detachment
}

\author{
GUOMIN ZHU ${ }^{1,2}$, MARIA SUSHKO ${ }^{2}$, JOHN LORING ${ }^{2}$, \\ BENJAMIN LEGG ${ }^{2}$, XIAOPENG HUANG ${ }^{2}$, MIAO SONG ${ }^{2}$, \\ KEVIN ROSSO $^{2}$ AND JIM DE YOREO ${ }^{2,3}$ \\ ${ }^{1} 2500$ George Washington Way \\ ${ }^{2}$ Pacific Northwest National Laboratory \\ ${ }^{3}$ University of Washington \\ Presenting Author: zhuguomin520520@gmail.com
}

Crystallization by particle attachment (CPA), which is a common mechanism of colloidal crystallization resulting in hierarchical morphologies, has been both exploited to create nanomaterials with unique, emergent properties and implicated in the development of complex mineral textures. Oriented attachment (OA), a form of CPA in which crystalline primary particles align and attach along specific crystallographic directions, produces structures - typically referred to as mesocrystals - that diffract like single crystals, even though the constituent particle domains are still discernable. While the existence of mesocrystals has been well documented in a wide range of crystal systems and individual particle attachment events have been directly visualized, the mechanism by which these seemingly random events lead to well-defined, self-similar morphologies remains a mystery, as does the role of organic ligands, which are ubiquitous in nanoparticle systems. Combining in situ TEM at $80^{\circ} \mathrm{C}$ with "freeze-and-look" TEM using indexed grids, we tracked formation of hematite (Hm) mesocrystals in the presence of oxalate and interpreted the results using classical density functional theory. The results show that formation of isolated $\mathrm{Hm}$ particles rarely occurs. However, once formed, interfacial gradients created by hematite-bound oxalate drive new hematite particles to repeatedly nucleate about $2 \mathrm{~nm}$ away from the new interface and then immediately undergo OA. Because $\mathrm{Hm}$ nucleation rates are statistically deterministic and direction-specific, the resulting mesocrystals are self-similar. In addition, we investigated the dissolution behavior of the resulting $\mathrm{Hm}$ mesocrystals. In contrast to the conventional picture of crystal dissolution by monomer detachment, we found a surprising dissolution behavior involving detachment of particles followed by their rapid dissolution. The resulting dissolution rates were found to greatly exceed those of faceted single hematite crystals, even after normalization of surface area. Using ex-situ HRTEM to gain atomic-resolution information about the pre- and post-dissolution structures, we identified misalignments and dislocations at the boundaries between the original nanoparticles in the branches of the mesocrystals. An analytical model that incorporates the effects of local curvature and strain on dissolution rate provides a rationale for the observed dissolution behavior.

Reference:

[1] Zhu, G., Sushko, M.L. et al. Nature 590, 416-422 (2021). 